This is the peer reviewed version of the following article:

Faillie, J. , Filion, K. B., Patenaude, V. , Ernst, P. and Azoulay, L. (2015), Dipeptidyl peptidase-4 inhibitors and the risk of community-acquired pneumonia in patients with type 2 diabetes. Diabetes Obes Metab, 17: 379385. doi:10.1111/dom.12431

which has been published in final form

https://onlinelibrary.wiley.com/doi/full/10.1111/dom.12431.

This article may be used for non-commercial purposes in accordance with Wiley Terms and Conditions for Use of Self-Archived Versions. 


\title{
Dipeptidyl peptidase-4 inhibitors and the risk of community-acquired pneumonia in patients with type 2 diabetes
}

\author{
J.-L. Faillie ${ }^{1,2,3}$, K. B. Filion ${ }^{1,4,5}$, V. Patenaude ${ }^{1,4,5}$, P. Ernst ${ }^{1}$ \& L. Azoulay ${ }^{1,6}$ \\ ${ }^{1}$ Centre for Clinical Epidemiology, Lady Davis Institute, Jewish General Hospital, Montreal, Quebec, Canada \\ ${ }^{2}$ Department of Pharmacoepidemiology, INSERM U1027, Faculty of Medicine, Paul Sabatier University, Toulouse, France \\ ${ }^{3}$ Department of Medical Pharmacology and Toxicology, Pharmacovigilance Regional Center, CHRU Montpellier University Hospital, Montpellier, France \\ ${ }^{4}$ Department of Epidemiology, Biostatistics, and Occupational Health, McGill University, Montreal, Quebec, Canada \\ ${ }^{5}$ Division of Clinical Epidemiology, Department of Medicine, McGill University, Montreal, Quebec, Canada \\ ${ }^{6}$ Department of Oncology, McGill University, Montreal, Quebec, Canada
}

Aims: To determine whether the use of dipeptidyl peptidase-4 (DPP-4) inhibitors is associated with an increased risk of community-acquired pneumonia. Methods: The UK Clinical Practice Research Datalink and the Hospital Episodes Statistics database were used to conduct a nested case-control analysis within a cohort of new users of antidiabetic drugs between 2007 and 2012. Incident cases of hospitalization for community-acquired pneumonia were matched with up to 20 controls on age, duration of treated diabetes, calendar year and duration of follow-up. Conditional logistic regression models were used to estimate the odds ratios (ORs) and 95\% confidence intervals (Cls) for hospitalization for community-acquired pneumonia associated with current use of DPP-4 inhibitors compared with current use of two or more oral antidiabetic drugs.

Results: The cohort included 49653 patients, of whom 562 were hospitalized for community-acquired pneumonia during follow-up (incidence rate 5.2/1000 person-years). Compared with current use of two or more oral antidiabetic drugs, current use of DPP-4 inhibitors was not associated with an increased risk of hospitalized community-acquired pneumonia overall (adjusted OR 0.80,95\% Cl 0.50-1.29) or according to duration of use ( $\mathrm{p}$ for trend $=0.57$ ).

Conclusions: The use of DPP-4 inhibitors was not associated with an increased risk of hospitalization for community-acquired pneumonia. Additional research is needed to assess the association between these drugs and other serious infections.

Keywords: database research, DPP-IV inhibitor, pharmaco-epidemiology, type 2 diabetes

Date submitted 10 December 2014; date of first decision 18 December 2014; date of final acceptance 2 January 2015

\section{Introduction}

Dipeptidyl peptidase-4 (DPP-4) inhibitors are a class of oral hypoglycaemic agents used to treat type 2 diabetes. These drugs increase glucagon like peptide-1 (GLP-1) endogeneous levels by inhibiting the DPP-4 enzyme (also known as CD26), which in turn increases glucose-stimulated insulin secretion and decreases glucagon release [1]. In addition to its role in glucose regulation, the DPP-4 enzyme has been shown to have pleiotropic effects [2]. It is expressed on the surface of leukocytes and stimulates inflammatory immune responses by modifying the production of several cytokines [2-5]. Consequently, it is hypothesized that DPP-4 inhibitors could alter the immune response and thus may play a role in the occurrence of infections. This hypothesis is supported by meta-analyses of randomized controlled trials and an adverse event database analysis, which found an association between DPP-4 inhibitors and an increased risk of infections, such as those of the respiratory and urinary tracts [6-8]. In addition, a meta-analysis of eight randomized controlled trials found a $29 \%$ increased risk of any infection with the DPP-4 inhibitor sitagliptin [9].

Correspondence to: Laurent Azoulay, PhD, Centre for Clinical Epidemiology, Lady Davis Institute, Jewish General Hospital, Montreal, Quebec H3T 1E2, Canada.

E-mail: laurent.azoulay@mcgill.ca
Lower respiratory tract infections, such as communityacquired pneumonia, are common in patients with diabetes [10], and are associated with a high mortality [11-14]. Although the association between DPP-4 inhibitors and infections in general has been studied previously, their effects on the risk of community-acquired pneumonia remain poorly understood. We therefore conducted a population-based study to determine, in a real-world setting, whether the use of DPP-4 inhibitors was associated with an increased risk of community-acquired pneumonia in a large population-based cohort of patients with type 2 diabetes.

\section{Materials and Methods}

\section{Data Sources}

The present nested case-control analysis was conducted by linking the UK Clinical Practice Research Datalink (CPRD) and the Hospital Episodes Statistics (HES) database. The CPRD is used to record demographic characteristics, diagnoses and drug prescriptions issued by general practictioners for $>13$ million individuals in 680 general practices [15]. Diagnoses and procedures recorded in the CPRD are based on the Read classification, and prescriptions written by general practitioners are based on the UK Prescription Pricing 
Authority Dictionary. Data collected in the CPRD have been validated and are of high quality [16]. Since 1997, the English CPRD practices can be linked to the HES database, which collects information on dates of hospital admissions, procedures and discharge diagnoses [coded using the International Classification of Diseases, 10th revision (ICD-10)].

The study protocol was approved by the Independent Scientific Advisory Committee of the CPRD (protocol number 13_034RA2) and by the Research Ethics Board of the Jewish General Hospital, Montreal, Canada.

\section{Study Population}

Base Cohort. All patients aged $\geq 18$ years and newly treated with a non-insulin antidiabetic drug (metformin, sulphonylureas, prandial glucose regulators, thiazolidinediones, acarbose, DPP-4 inhibitors and GLP-1 analogues) between 1 January 1988 and 31 March 2012 were identified. We excluded patients initially treated with insulin, as these represent patients with type 1 diabetes or an advanced type 2 diabetes. We also excluded patients with a history of polycystic ovarian syndrome, as this is another known indication for metformin. All patients were required to have at least 1 year of baseline medical history in the CPRD before their first non-insulin prescription.

Study Cohort. Within the base cohort defined above, we assembled a study cohort composed of all patients who began taking a new antidiabetic drug on or after 2007 (the year the first DPP-4 inhibitor, sitagliptin, was licensed in the UK). These patients included those newly treated with a non-insulin antidiabetic drug, as well as those who switched or added-on an antidiabetic drug not previously used in the patient's treatment history. Study cohort entry was defined by the date of this new prescription. We excluded all patients diagnosed with a lower respiratory tract infection, as well as those hospitalized for any cause (with a length of stay $\geq 2$ days) in the 30 days before cohort entry, to ensure that cases of pneumonia were community-acquired. We also excluded patients with a history of any cancer (except non-melanoma skin cancer) at any time before cohort entry and those with prescriptions for antituberculosis drugs in the year before cohort entry.

All patients meeting the study inclusion criteria were followed until a hospitalization for community-acquired pneumonia or censoring attributable to any hospitalization lasting $\geq 2$ days, death from any cause, end of registration with the general practice, end of HES linkage or end of the study period (31 March 2012), whichever came first.

\section{Case-Control Selection}

A nested case-control analysis was performed within the study cohort defined above. Cases consisted of patients hospitalized for community-acquired pneumonia, defined as a hospitalization for pneumonia (ICD-10 codes: B01.2, B05.2, B20.6, B25.0, J10.0, J11.0, J12-J18, J85.1, U04, U04.9) recorded in the HES database in primary or secondary position within the first 2 days of the admission. Our case definition was restricted to the first 2 days of admission to ensure that cases were community-acquired and not nosocomial pneumonia. Only hospitalizations with a length of stay $>1$ day were included as cases, except for patients who died on their admission date. The index date was defined as the case's date of hospital admission.

Each case was randomly matched, using risk-set sampling, with up to 20 control subjects selected from patients in the cohort [i.e. who at the time of the case's event (or index) date, were still at risk of developing the outcome]. Controls were matched to cases on age ( \pm 1 year), duration of treated diabetes (time from the first non-insulin antidiabetic prescription to cohort entry \pm 90 days), year of study cohort entry and duration of follow-up. For 17 cases, the matching criteria were relaxed using larger calipers for year of study cohort entry ( \pm 1 year), age ( \pm 5 years) and duration of treated diabetes ( \pm 90 days). The controls were assigned the index date of their respective case.

\section{Exposure Definition}

Exposure to antidiabetic drugs was assessed at the index date and defined hierarchically according to the following six mutually exclusive categories: (i) current use of a DPP-4 inhibitor, such as sitagliptin, saxagliptin, vildagliptin or linagliptin; (ii) current use of a GLP-1 analogue, such as exenatide or liraglutide; (iii) current use of insulin; (iv) current use of two or more oral antidiabetic drugs; (v) current use of an oral antidiabetic agent in monotherapy; and (vi) no current use of an antidiabetic agent at index date. For all categories above, current use was defined by a prescription duration plus a 30-day grace period that overlapped with the index date. The reference category for all analyses consisted of patients currently using two or more oral antidiabetic agents.

\section{Potential Confounders}

All models were adjusted for the following potential confounders measured at study cohort entry: sex, body mass index (BMI), glycated haemoglobin (HbAlc) concentration, excessive alcohol use (based on diagnoses for alcohol-related disorders, such as alcoholism, alcoholic cirrhosis of the liver, alcoholic hepatitis and hepatic failure), smoking, number of physician visits in the previous year, history of pulmonary comorbidities (lower respiratory tract infection, asthma, chronic obstructive pulmonary disease, bronchiectasis; assessed in the year before cohort entry), diabetic arterial complications (retinopathy, neuropathy, nephropathy, peripheral arteriopathy, myocardial infarction, stroke), use of antidiabetic drugs (metformin, sulphonylureas, thiazolidinediones, insulins and other agents; assessed in the year before cohort entry), use of immunosuppressive agents, inhaled bronchodilators, inhaled corticosteroids, non-topical antibiotics, non-topical corticosteroids (assessed in the year before cohort entry), and use of influenza or pneumococcal vaccines (assessed in the year before cohort entry). Variables with missing information were coded as 'unknown' [17].

\section{Statistical Analysis}

Primary Analysis. Descriptive statistics were used to summarize the characteristics of the cases and matched controls. A crude incidence rate, with $95 \%$ confidence intervals (CIs) based 
on the Poisson distribution, was calculated by dividing the number of patients hospitalized for community-acquired pneumonia over the person-time at risk. Conditional logistic regression models were used to estimate odds ratios (ORs) with $95 \%$ CIs of hospitalized community-acquired pneumonia associated with current use of DPP-4 inhibitors, compared with current use of two or more oral antidiabetic agents. This analysis was considered to be the primary analysis.

Secondary Analyses. We performed three secondary analyses. First, we evaluated whether the risk varied with duration of treatment among current users of DPP-4 inhibitors. Patients were considered continuously exposed if the duration of one prescription overlapped the date of the subsequent prescription, allowing for a 30-day grace period between two successive, non-overlapping prescriptions. Second, we analysed the risk of hospitalized community-acquired pneumonia for each type of DPP-4 inhibitor. Third, we assessed whether duration of treated diabetes modified the association between current use of DPP-4 inhibitors and hospitalized community-acquired pneumonia. For that analysis, an interaction term between exposure and duration of treated diabetes was included in the regression model.

Sensitivity Analyses. We conducted seven sensitivity analyses. First, we repeated the primary analysis using grace periods of 0 and 90 days. Second, to assess the choice of our exposure groups, we compared the current use of DPP-4 inhibitors with all possible antidiabetic drug combinations that did not include a DPP-4 inhibitor. Third, we conducted analyses using a subcohort of patients who did not receive insulin (a marker of increased severity of diabetes) or thiazolininediones (which have previously been associated with pneumonia) [18] before cohort entry and for whom we also censored the follow-up time at the first prescription of insulin or thiazolininediones. Fourth, we repeated our primary analysis using the combination of metformin and sulphonylureas as the reference group. Fifth, the hierarchical classification of exposure was reordered, considering exposure to insulin first, thiazolininediones second and then DPP-4 inhibitors. Sixth, to assess the robustness of our outcome defintion, we repeated our analyses with our case series restricted to those identified in the HES database by an ICD-10 code in primary position only. Finally, to study the effect of the time of covariate measurement, we repeated our analyses with models adjusted for potential confounders measured at index date instead of cohort entry. All analyses were conducted using sas version 9.3 (SAS Institute, Cary, NC, USA).

\section{Results}

\section{Primary Analysis}

The study cohort included 49653 new users of antidiabetic agents as of 2007 (Figure 1). The mean [standard deviation

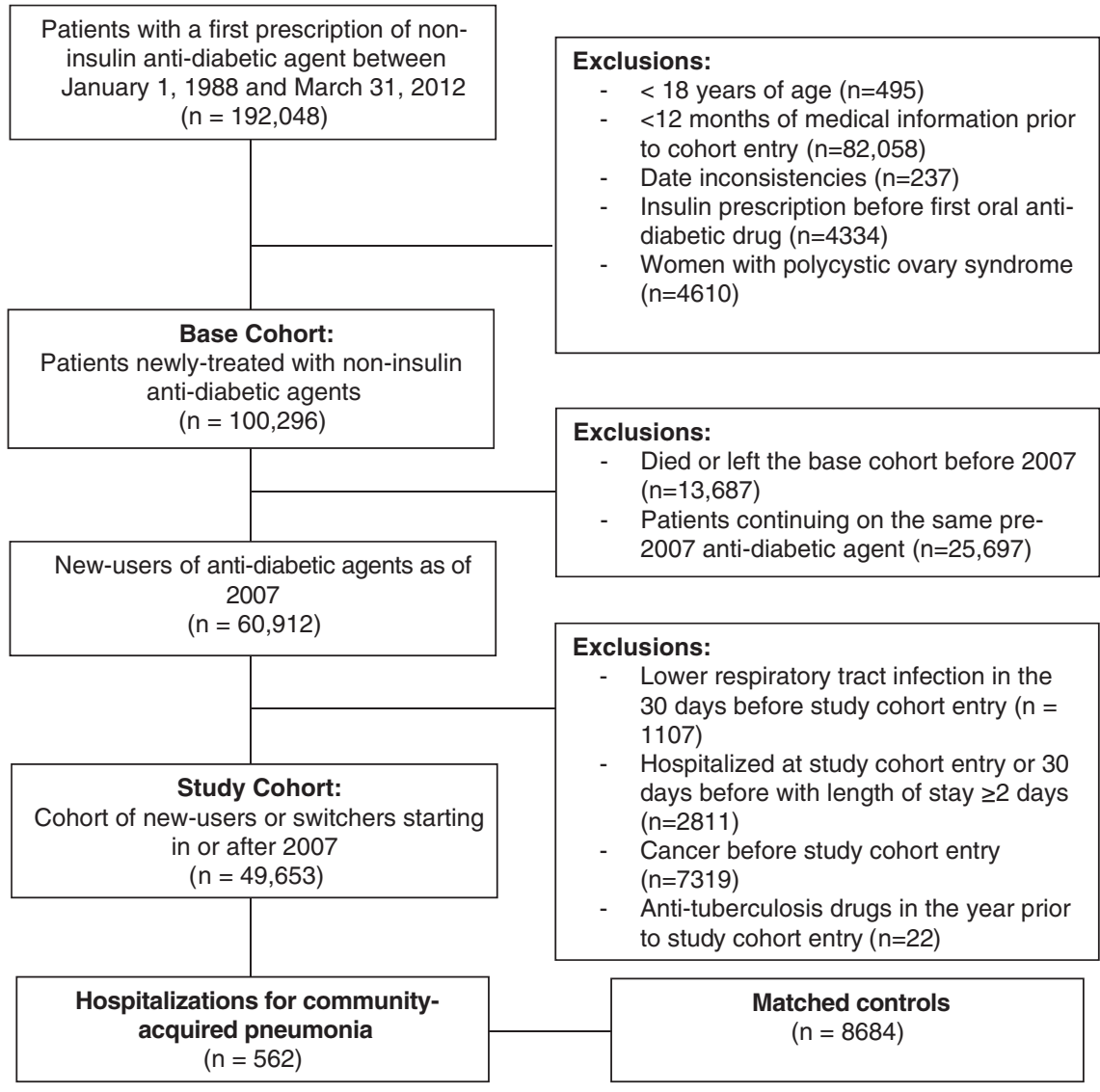

Figure 1. Study flow chart. 
Table 1. Baseline characteristics of cases of hospitalized communityacquired pneumonia and matched controls.

\begin{tabular}{|c|c|c|}
\hline Baseline characteristics & $\begin{array}{l}\text { Cases } \\
\mathrm{n}=562\end{array}$ & $\begin{array}{l}\text { Controls } \\
\mathrm{n}=8684\end{array}$ \\
\hline Age, years, mean (s.d.) & $68.4(13.1)$ & $68.4(13.1)$ \\
\hline Males, n (\%) & $319(56.8)$ & $4725(54.1)$ \\
\hline $\begin{array}{l}\text { Duration of treated diabetes, years, } \\
\text { mean (s.d.) }\end{array}$ & $1.9(3.3)$ & $1.9(3.3)$ \\
\hline \multicolumn{3}{|l|}{ Year of cohort entry, n (\%) } \\
\hline 2007 & $183(32.6)$ & $2821(32.2)$ \\
\hline 2008 & $146(26.0)$ & $2172(27.0)$ \\
\hline 2009 & $110(19.6)$ & $1718(19.0)$ \\
\hline 2010 & $80(14.2)$ & $1282(14.3)$ \\
\hline 2011 & S & $671(7.3)$ \\
\hline 2012 & S & $20(0.2)$ \\
\hline \multicolumn{3}{|l|}{ Body mass index, $\mathrm{n}(\%)$} \\
\hline$\leq 25 \mathrm{~kg} / \mathrm{m}^{2}$ & $106(18.9)$ & $1209(15.3)$ \\
\hline $26-30 \mathrm{~kg} / \mathrm{m}^{2}$ & $173(30.8)$ & $2885(33.1)$ \\
\hline$>30 \mathrm{~kg} / \mathrm{m}^{2}$ & $265(47.2)$ & $4364(49.5)$ \\
\hline Unknown & $18(3.2)$ & $226(2.2)$ \\
\hline Excessive alcohol use, n (\%) & $111(19.8)$ & $1021(11.8)$ \\
\hline \multicolumn{3}{|l|}{ Smoking status, $\mathrm{n}(\%)$} \\
\hline Ever & $415(73.8)$ & $5242(59.9)$ \\
\hline Never & $\mathrm{S}$ & $3394(39.7)$ \\
\hline Unknown & S & $48(0.4)$ \\
\hline $\begin{array}{l}>4 \text { physician visits in the year before } \\
\text { cohort entry, } \mathrm{n}(\%)\end{array}$ & $556(98.9)$ & $8366(97.1)$ \\
\hline \multicolumn{3}{|l|}{ HbAlc categories, n (\%) } \\
\hline$\leq 7 \%(53 \mathrm{mmol} / \mathrm{mol})$ & $78(13.9)$ & $1207(12.9)$ \\
\hline $7.1-8 \%(54-64 \mathrm{mmol} / \mathrm{mol})$ & $150(26.7)$ & $2677(31.2)$ \\
\hline$>8 \%(64 \mathrm{mmol} / \mathrm{mol})$ & $240(42.7)$ & $3345(42.7)$ \\
\hline Unknown & $94(16.7)$ & $1455(13.3)$ \\
\hline
\end{tabular}

HbA1c, glycated haemoglobin; S, suppressed as at least one cell has a count $<5$; s.d., standard deviation.

(s.d.)] age of the study subjects was 59.6 (13.3) years, 58.2\% were males, and the mean (s.d.) duration of treated diabetes before entry to the study cohort was 1.5 (3.0) years. The baseline characteristics of the study cohort are summarized in Table S1. The study cohort was followed for a mean (s.d.) of 2.2 (1.5) years, generating a total of 106718 person-years of follow-up. Overall, a total of 562 patients were hospitalized for community-acquired pneumonia during follow-up, generating an incidence rate of 5.2/1000 (95\% CI 4.9-5.7) person-years.

The characteristics of the cases and matched controls are shown in Table 1. Other baseline conditions and medications are shown in Table S2. Cases and controls were similar in terms of BMI, HbAlc concentration, use of oral antidiabetic agents and microangiopathic complications of type 2 diabetes (retinopathy, neuropathy and nephropathy). By contrast, compared with controls, cases were more likely to have smoked, to have used alcohol excessively, and to have a history of myocardial infarction, stroke, peripheral arteriopathy and pulmonary comorbidities (lower respiratory tract infection, asthma, chronic obstructive pulmonary disease, bronchiectasis). For both cases and matched controls, the mean (s.d.) duration of follow-up was 1.5 (1.2) years.

The results of the primary and secondary analyses are shown in Table 2. After adjustment for potential confounders, current use of DPP-4 inhibitors was not associated with an increased risk of hospitalized community-acquired pneumonia, when compared with current use of two or more oral antidiabetic agents (adjusted OR 0.80, 95\% CI 0.50-1.29). There was no evidence of a duration-response relationship, with no OR found to be statistically significant and all ORs under the null value ( $\mathrm{p}$ for trend $=0.57$ ). In terms of individual DDP-4 inhibitor types, no single agent was associated with an increased risk of hospitalized community-acquired pneumonia. Finally, there was no evidence that the association varied with duration of treated diabetes ( $<5$ years, adjusted OR $0.79,95 \%$ CI $0.44-1.41 ; \geq 5$ years, adjusted OR $0.91,95 \%$ CI $0.40-2.09$; $\mathrm{p}$ for interaction $=0.17$ ).

\section{Sensitivity Analyses}

As with our primary analyses, none of the sensitivity analyses revealed an association between the use of DPP-4 inhibitors and the risk of hospitalized community-acquired pneumonia (Tables S3-S9).

\section{Discussion}

The results of this large population-based study indicate that the use of DPP-4 inhibitors when compared with the use of other oral antidiabetic agents is not associated with an increased risk of hospitalized community-acquired pneumonia. Similar null findings were observed in secondary analyses, and the results remained robust in a number of sensitivity analyses. To date, our study is the first observational study using a large population-based cohort to assess the association between DPP-4 inhibitors and the risk of community-acquired pneumonia.

Our null findings contrast with experimental data suggesting an immunomodulatory effect of DPP-4 inhibitors. Indeed, in addition to the GLP-1, other regulatory peptides, including cytokines and chemokines, are substrates of DPP-4/CD26 [2-4]. Thus, DPP-4/CD26 is thought to enhance T-cell activation [19], and studies have suggested that DPP-4 inhibition suppresses DNA synthesis of mononucleocytes and T-cells in vitro [4]. This leads to the upregulation of the immunosuppressive cytokine transforming growth factor- $\beta 1$ and the inhibition of T-cells in mice [5]; however, these findings are not supported by a recent in vitro and in vivo study [20]. In addition, there are mechanistic differences between sitagliptin and vildagliptin with regard to markers of oxidative stress and systemic inflammation [20-22], such that differential modification of immune responses within the class of DPP-4 inhibitors cannot be excluded. In the present study, with 22 of 25 cases exposed to sitagliptin, there were insufficient data to conclusively examine the risk of hospitalized community-acquired pneumonia by type of DPP-4 inhibitor.

Within the World Health Organization pharmacovigilance database (VigiBase), the reporting of infections was higher for patients using DPP-4 inhibitors than for users of metformin (reporting OR 2.3, 95\% CI 1.9-2.7), with a stronger association for upper respiratory tract infections (reporting OR 12.3, 95\% CI 8.6-17.5) [8]; however, given the numerous biases 
Table 2. Risk of hospitalization for community-acquired pneumonia according to current exposure to antidiabetic agents.

\begin{tabular}{|c|c|c|c|c|}
\hline Current exposure* & $\begin{array}{l}\text { Cases } \\
\mathrm{n}=562\end{array}$ & $\begin{array}{l}\text { Controls } \dagger \\
\mathbf{n}=\mathbf{8 6 8 4}\end{array}$ & Crude OR (95\% CI) & Adjusted OR $\ddagger(95 \%$ CI $)$ \\
\hline$\geq 2$ oral anti-diabetic agents, $n(\%)$ & $149(26.5)$ & $1708(19.7)$ & 1.00 (reference) & 1.00 (reference) \\
\hline DPP-4 inhibitors, $\mathrm{n}(\%)$ & $25(4.5)$ & $336(3.9)$ & $0.77(0.49-1.23)$ & $0.80(0.50-1.29)$ \\
\hline \multicolumn{5}{|c|}{ Duration of DPP-4 inhibitor use $\$$ n (\%) } \\
\hline $1-107$ days & $9(1.6)$ & $111(1.3)$ & $0.83(0.40-1.75)$ & $0.95(0.44-2.04)$ \\
\hline 108-304 days & $8(1.4)$ & $111(1.3)$ & $0.88(0.40-1.94)$ & $0.79(0.35-1.77)$ \\
\hline$\geq 305$ days & $8(1.4)$ & $114(1.3)$ & $0.64(0.30-1.39)$ & $\begin{array}{l}0.70(0.32-1.55) \\
p \text { for trend }=0.57\end{array}$ \\
\hline \multicolumn{5}{|l|}{ Types of DPP-4 inhibitors, $\mathrm{n}(\%)$} \\
\hline Sitagliptin & $22(3.9)$ & $281(3.2)$ & $0.83(0.51-1.36)$ & $0.88(0.53-1.46)$ \\
\hline Vildagliptin & $\mathrm{S}$ & $39(0.5)$ & $0.43(0.10-1.90)$ & $0.45(0.10-2.00)$ \\
\hline Saxagliptin & $\mathrm{S}$ & $\mathrm{S}$ & $0.83(0.11-6.54)$ & $0.57(0.06-5.02)$ \\
\hline Linagliptin & $0(0.0)$ & $\mathrm{S}$ & - & - \\
\hline
\end{tabular}

CI, confidence interval; DPP-4, dipeptidyl peptidase-4; OR, odds ratio; S, suppressed as at least one cell has a count $<5$.

${ }^{\star}$ Current users of glucagon-like peptide 1 analogues, insulins, single oral antidiabetic drugs, and non-current users of antidiabetic drugs are not shown in the table, but were considered in the regression model for proper estimation of treatment effects (representing 388 cases and 6640 controls).

$\dagger$ Cases and controls were matched on age, duration of treated diabetes, calendar year of study cohort entry, and duration of follow-up.

†Adjusted for sex, body mass index, number of antidiabetic drugs ever used, glycated haemoglobin concentration, excessive alcohol use, smoking, history of lower respiratory tract infections, use of antidiabetic drugs (metformin, sulphonylureas, thiazolidinediones, insulins and other agents), more than four physician visits in the year before cohort entry, asthma, chronic obstructive pulmonary disease or bronchiectasis, use of immunosuppressive agents, inhaled bronchodilators, inhaled corticosteroids, non-topical antibiotics, oral corticosteroids, influenza or pneumococcal vaccines.

$\S$ Duration categories based on the tertile distribution among controls.

involved in reporting adverse events, the conclusions that can be drawn from adverse events databases are limited [23]. To date, no other pharmacoepidemiological study has investigated pneumonia as an adverse outcome. A population-based cohort study of 72738 new users of oral antidiabetic drugs between 2004 and 2009 retrieved from a large US insurance claims database, did not find any significant association between sitagliptin and upper respiratory tract infections; lower respiratory tract infections were not examined [24]. Interestingly, another recent cohort study using US insurance claims data from 2005 to 2012 provided evidence of an immunomodulating effect of DPP-4 inhibitors: the risks of incident rheumatoid arthritis and other autoimmune diseases were lower in users of DPP-4 inhibitors compared with non-users [25]. In 2008, a meta-analysis of randomized controlled trials performed by the Cochrane Collaboration, which included 3589 patients, showed a statistically significant $29 \%$ increase in all-cause infections associated with sitagliptin (no association was found for vildagliptin) [9]. In addition, two meta-analyses reported an increased risk of nasopharyngitis and of urinary tract infection [6,7]. By contrast, several recent meta-analysis of randomized controlled trials concluded that there was no increased risk of pneumonia with sitagliptin when compared with placebo or active comparators [26-28]. DPP-4 inhibitor use was not associated with an increased incidence of respiratory infections in three pooled safety analyses of randomized clinical trials (generally $<2$ years in duration) of sitagliptin, saxagliptin and linagliptin, which included 10246,9156 and 3572 patients, respectively [29-31].

The strengths of the present study include the use of a large population-based cohort for which data sources have been shown to be of high quality and provide the necessary information to account for important potential confounders
(BMI, excessive alcohol use, HbA1c). The use of a base cohort of patients with diabetes followed for up to 25 years allows a comparison of patients at similar points in the natural history of the disease. We chose to focus on hospitalized community-acquired pneumonia because it is common, is associated with significant morbidity and mortality [32] and has been previously used in the HES database [33,34]. Another strength was our choice of comparator. Because DPP-4 inhibitors are used as second-/third-line therapy and as there is no pharmaceutical class available or suitable for a direct comparison, we considered the group of patients who were prescribed two or more oral antidiabetic agents as the most clinically relevant comparator group, limiting potential confounding by indication.

The present study has some potential limitations. First, as we used prescriptions by general practitioners to define drug exposure, we may have underestimated the exposure to DPP-4 inhibitors because prescriptions written by specialists were not included; however, all patients entered the study cohort based on a prescription issued by their general practitioner, and both our exposure and comparison groups consisted of patients currently exposed to antidiabetic drugs prescribed by general practitioners, suggesting that this was unlikely to have biased our results. Some misclassification of exposure is also possible as the CPRD records prescriptions that are written rather than filled and does not contain information regarding patient adherence, although there is no reason to believe that any potential lack of adherence was different between the exposure groups. Our outcome definition, restricted to hospitalized community-acquired pneumonia, underestimated the overall incidence of community-acquired pneumonia because of the exclusion of non-hospitalized patients or those with only 1 day of hospitalization. Our overall incidence rate of hospitalized 
community-acquired pneumonia of 5.22/1000 person-years is less than that found in another study of patients with diabetes using the CPRD [10]. In the latter study, the rate of community-acquired pneumonia was $10.3 / 1000$ person-years, and up to $81.4 \%$ of the cases were hospitalized. The difference is probably attributable to the fact that this previous study only included patients aged $\geq 65$ years and also included patients with type 1 diabetes [10]. Another previous study of an administrative database from Ontario, Canada found a much higher rate of pneumonia in patients with diabetes (49.2/1000 person-years), but was based on an older population, where the outcome definition also included hospital-acquired pneumonia as well as outpatient claims for pneumonia (which could possibly include other respiratory tract infections, such as bronchitis) [35]. Although respiratory tract infections are relatively frequent in patients with diabetes, hospitalization for community-acquired pneumonia is much less common, limiting our statistical power for secondary analyses. Overall, the present study cannot exclude a modest increase (up to 29\%) as well as a $50 \%$ decrease in the incidence of pneumonia. Finally, because of the observational nature of the present study and despite the adjustment for important risk factors, the possible effects of residual confounding must be considered when interpreting our results.

In summary, the present study does not support an increased risk of hospitalization for community-acquired pneumonia with the use of DPP-4 inhibitors among patients with type 2 diabetes. Nevertheless, more research is needed to determine whether DPP-4 inhibitors are associated with the risk of other serious infections.

\section{Acknowledgements}

J.-L. F. is the recipient of a grant from the Sociéte Francophone du Diabète, and K. F. holds a Canadian Institutes of Health Research (CIHR) New Investigator Award. This study was funded in part by research grants from the CIHR, and the Canada Foundation for Innovation.

\section{Conflict of Interest}

The authors have no conflict of interest to declare.

J.-L. F. contributed to the study conception and design, drafted the manuscript, and contributed to the interpretation of data, and critical revision of the manuscript for important intellectual content. K. B. F., P. E. and L. A. contributed to the study conception and design, the conduct of the statistical analyses, interpretation of data and critical revision of the manuscript for important intellectual content. V. P. contributed to the statistical analyses, interpretation of data and critical revision of the manuscript for important intellectual content. L. A. is the guarantor of this work and, as such, had full access to all the data in the study and takes responsibility for the integrity of the data and the accuracy of the data analysis.

\section{Supporting Information}

Additional Supporting Information may be found in the online version of this article:
Table S1. Baseline characteristics of the study cohort population.

Table S2. Baseline conditions and medications of cases hospitalized for community-acquired pneumonia and matched controls.

Table S3. Risk of hospitalization for community-acquired pneumonia with current exposure to antidiabetic agents, according to varying grace period durations.

Table S4. Risk of hospitalization for community-acquired pneumonia with current exposure to antidiabetic agents in the subcohort of patients who did not receive insulin or thiazolininediones before cohort entry and censoring those who received insulin or thiazolininediones during follow-up.

Table S5. Risk of hospitalization for community-acquired pneumonia with current exposure to dipeptidyl peptidase- 4 (DPP-4) inhibitors compared with all other possible antidiabetic combinations which did not include a DPP-4 inhibitor.

Table S6. Risk of hospitalization for community-acquired pneumonia with current exposure to dipeptidyl peptidase- 4 inhibitor compared with the combination of metformin and sulfonylureas.

Table S7. Risk of hospitalization for community-acquired pneumonia with current exposure to antidiabetic agents when changing the hierarchical classification of exposure considering exposure to insulin first, thiazolininediones second, and then dipeptidyl peptidase- 4 inhibitors.

Table S8. Risk of hospitalization for community-acquired pneumonia with current exposure to antidiabetic agents when hospitalization for community-acquired pneumonia was defined in the Hospital Episode Statistics database by a ICD-10 in primary position only.

Table S9. Risk of hospitalization for community-acquired pneumonia with current exposure to antidiabetic agents when covariates were measured at index date instead of cohort entry.

\section{References}

1. Drucker DJ. Biological actions and therapeutic potential of the glucagon-like peptides. Gastroenterology 2002; 122: 531-544.

2. Mentlein R. Dipeptidyl-peptidase IV (CD26)-role in the inactivation of regulatory peptides. Regul Pept 1999; 85: 9-24.

3. Ou X, O'Leary HA, Broxmeyer HE. Implications of DPP4 modification of proteins that regulate stem/progenitor and more mature cell types. Blood 2013; 122: 161-169.

4. Reinhold D, Biton A, Goihl A et al. Dual inhibition of dipeptidyl peptidase IV and aminopeptidase $\mathrm{N}$ suppresses inflammatory immune responses. Ann N Y Acad Sci 2007; 1110: 402-409.

5. Steinbrecher A, Reinhold D, Quigley L et al. Targeting dipeptidyl peptidase IV (CD26) suppresses autoimmune encephalomyelitis and up-regulates TGF-beta 1 secretion in vivo. J Immunol 2001; 166: 2041-2048.

6. Monami M, lacomelli I, Marchionni N, Mannucci E. Dipeptydil peptidase-4 inhibitors in type 2 diabetes: a meta-analysis of randomized clinical trials. Nutr Metab Cardiovasc Dis 2010; 20: 224-235.

7. Amori RE, Lau J, Pittas AG. Efficacy and safety of incretin therapy in type 2 diabetes: systematic review and meta-analysis. JAMA 2007; 298: 194-206.

8. Willemen MJ, Mantel-Teeuwisse AK, Straus SM, Meyboom RH, Egberts TC, Leufkens HG. Use of dipeptidyl peptidase-4 inhibitors and the reporting of infections: a disproportionality analysis in the World Health Organization VigiBase. Diabetes Care 2011; 34: 369-374. 
9. Richter B, Bandeira-Echtler E, Bergerhoff K, Lerch CL. Dipeptidyl peptidase-4 (DPP-4) inhibitors for type 2 diabetes mellitus. Cochrane Database Syst Rev 2008; 16: CD006739.

10. McDonald HI, Nitsch D, Millett ER, Sinclair A, Thomas SL. New estimates of the burden of acute community-acquired infections among older people with diabetes mellitus: a retrospective cohort study using linked electronic health records. Diabet Med 2014; 31: 606-614.

11. Wunderink RG, Waterer GW. Clinical practice. Community-acquired pneumonia. N Engl J Med 2014; 370: 543-551.

12. Fine MJ, Smith MA, Carson CA et al. Prognosis and outcomes of patients with community-acquired pneumonia. A meta-analysis. JAMA 1996; 275: 134-141.

13. Valdez R, Narayan KM, Geiss LS, Engelgau MM. Impact of diabetes mellitus on mortality associated with pneumonia and influenza among non-Hispanic black and white US adults. Am J Public Health 1999; 89: 1715-1721.

14. Yende $S$, van der Poll $T$, Lee $M$ et al. The influence of pre-existing diabetes mellitus on the host immune response and outcome of pneumonia: analysis of two multicentre cohort studies. Thorax 2010; 65: 870-877.

15. Garcia Rodriguez LA, Perez Gutthann S. Use of the UK General Practice Research Database for pharmacoepidemiology. Br J Clin Pharmacol 1998; 45 419-425

16. Herrett $E$, Thomas SL, Schoonen WM, Smeeth L, Hall AJ. Validation and validity of diagnoses in the General Practice Research Database: a systematic review. Br J Clin Pharmacol 2010; 69: 4-14.

17. Huberman M, Langholz B. Application of the missing-indicator method in matched case-control studies with incomplete data. Am J Epidemiol 1999; 150 1340-1345.

18. Singh S, Loke YK, Furberg CD. Long-term use of thiazolidinediones and the associated risk of pneumonia or lower respiratory tract infection: systematic review and meta-analysis. Thorax 2011; 66: 383-388.

19. De Meester I, Scharpe S, Lambeir AM. Dipeptidyl peptidases and related proteins: multifaceted markers and therapeutic targets. Clin Chem Lab Med 2009; 47 245-247.

20. Anz D, Kruger S, Haubner S, Rapp M, Bourquin C, Endres S. The dipeptidylpeptidase-IV inhibitors sitagliptin, vildagliptin and saxagliptin do not impair innate and adaptive immune responses. Diabetes Obes Metab 2014; 16: 569-572.

21. Miyagawa $K$, Kondo $T$, Goto $R$ et al. Effects of combination therapy with vildagliptin and valsartan in a mouse model of type 2 diabetes. Cardiovasc Diabetol 2013; 12: 160; DOI: 10.1186/1475-2840-12-160.

22. Rizzo MR, Barbieri M, Marfella R, Paolisso G. Reduction of oxidative stress and inflammation by blunting daily acute glucose fluctuations in patients with type
2 diabetes: role of dipeptidyl peptidase-IV inhibition. Diabetes Care 2012; 35 : 2076-2082.

23. Montastruc JL, Sommet $A$, Bagheri $H$, Lapeyre-Mestre M. Benefits and strengths of the disproportionality analysis for identification of adverse drug reactions in a pharmacovigilance database. Br J Clin Pharmacol 2011; 72: 905-908.

24. Eurich DT, Simpson S, Senthilselvan A, Asche CV, Sandhu-Minhas JK, McAlister FA. Comparative safety and effectiveness of sitagliptin in patients with type 2 diabetes: retrospective population based cohort study. BMJ 2013; 346: f2267.

25. Kim SC, Schneeweiss S, Glynn RJ, Doherty $M$, Goldfine $A B$, Solomon $D H$. Dipeptidyl peptidase-4 inhibitors in type 2 diabetes may reduce the risk of autoimmune diseases: a population-based cohort study. Ann Rheum Dis 2014; DOI: 10.1136/annrheumdis-2014-205216 [Epub ahead of print].

26. Engel SS, Round E, Golm GT, Kaufman KD, Goldstein BJ. Safety and tolerability of sitagliptin in type 2 diabetes: pooled analysis of 25 clinical studies. Diabetes Ther 2013; 4: 119-145.

27. Karagiannis $T$, Paschos $P$, Paletas $K$, Matthews DR, Tsapas A. Dipeptidyl peptidase-4 inhibitors for treatment of type 2 diabetes mellitus in the clinical setting: systematic review and meta-analysis. BMJ 2012; 344: e1369.

28. Goossen K, Graber S. Longer term safety of dipeptidyl peptidase-4 inhibitors in patients with type 2 diabetes mellitus: systematic review and meta-analysis. Diabetes Obes Metab 2012; 14: 1061-1072.

29. Williams-Herman D, Engel SS, Round E et al. Safety and tolerability of sitagliptin in clinical studies: a pooled analysis of data from 10,246 patients with type 2 diabetes. BMC Endocr Disord 2010; 10: 1-21.

30. Hirshberg $B$, Parker $A$, Edelberg $H$, Donovan $M$, lqbal N. Safety of saxagliptin: events of special interest in 9156 patients with type 2 diabetes mellitus. Diabetes Metab Res Rev 2014; 30: 556-569.

31. Schernthaner $G$, Barnett $A H$, Emser A et al. Safety and tolerability of linagliptin: a pooled analysis of data from randomized controlled trials in 3572 patients with type 2 diabetes mellitus. Diabetes Obes Metab 2012; 14: 470-478.

32. Fry AM, Shay DK, Holman RC, Curns AT, Anderson $\sqcup$. Trends in hospitalizations for pneumonia among persons aged 65 years or older in the United States, 1988-2002. JAMA 2005; 294: 2712-2719.

33. Filion KB, Chateau D, Targownik LE et al. Proton pump inhibitors and the risk of hospitalisation for community-acquired pneumonia: replicated cohort studies with meta-analysis. Gut 2013; 63: 552-558.

34. Skull SA, Andrews RM, Byrnes GB et al. ICD-10 codes are a valid tool for identification of pneumonia in hospitalized patients aged $>$ or $=65$ years. Epidemiol Infect 2008; 136: 232-240.

35. Shah BR, Hux JE. Quantifying the risk of infectious diseases for people with diabetes. Diabetes Care 2003; 26: 510-513. 